\title{
Effects of sevoflurane on apoptosis, BDNF and cognitive functions in neonatal rats
}

\author{
Ozer $\mathrm{AB}^{1}$, Ceribasi $\mathrm{S}^{2}$, Ceribasi $\mathrm{AO}^{2}$, Demirel $\mathrm{I}^{1}$, Bayar $\mathrm{MK}^{3}$, Ustundag $\mathrm{B}^{4}$, Ileri $\mathrm{A}^{1}$, Erhan $\mathrm{OL}^{1}$ \\ Department of Anesthesiology and Reanimation, Firat University, Faculty of Medicine, \\ Elazig, Turkey. abelinozer@gmail.com
}

\begin{abstract}
OBJECTIVE: To evaluate the early and late effects of sevoflurane on the neonatal brain.

BACKGROUND: Sevoflurane is the most used anaesthetics in neonatal subjects.

METHODS: The study included 7-day-old male Wistar-Albino rats $(n=30)$, which were divided into the two groups according to the anaesthetic received: sevoflurane (S) and control group (C). Half of each group was sacrificed six hours after anaesthesia (early, E) while the remaining subjects were sacrificed six weeks later (late, L). The serum brain-derived-neurotrophic factor (BDNF), brain BDNF and caspase-3 were evaluated. In addition, elevated plus arm test and Morris water test were performed in the late group.

RESULTS: BDNF levels were higher in the late groups than in the early ones $(p<0.05)$. BDNF levels in cerebral cortex were higher in the Group CE than in the Group CL and SL $(p<0.05)$. There was a significant negative correlation between serum BDNF and cortex BDNF levels $(p=0.003, r=-0.425)$. Cortex caspase 3 levels were significantly higher in the Groups SE and SL than in the Group CE and CL $(p<0.05)$. There was no significant difference between the groups in the terms of open arm index, locomotor activity and Morris water test. CONCLUSIONS: Although sevoflurane induced apoptosis, it didn't affect BDNF levels and showed no longterm negative effects on learning and anxiety in neonatal rats (Tab. 1, Fig. 3, Ref. 26). Text in PDF www.elis.sk. KEY WORDS: anesthesia, neonatal, apoptosis, BDNF.
\end{abstract}

\section{Introduction}

Brain-derived neurotrophic factor (BDNF) has important implications in the survival, growth and differentiation of existing neurons in the central and peripheral nervous system $(1,2)$. At the same time, BDNF was shown to be active in the hippocampus, cerebral cortex, cerebellum and basal forebrain, which are the areas that carry out vital functions such as learning, memory and thinking (3). BDNF shows its effect by the production of neurotransmitters, ion channel expression and by inducing longterm changes in synaptic composition $(4,5)$. In addition, BDNF enhances excitatory neurotransmission via its presynaptic and postsynaptic effects. Furthermore, it modulates excitatory and inhibitory synaptic transmission by gamma amino butyric acid (GABA)-A receptor-mediated inhibition of postsynaptic current. In immature brains, this effect occurs by an increase in GABAergic

${ }^{1}$ Department of Anesthesiology and Reanimation, Firat University, Faculty of Medicine, Elazig, Turkey, ${ }^{2}$ Department of Pathology, Firat University Faculty of Veterinary Medicine, Elazig, Turkey, ${ }^{3}$ Department of Anesthesiology and Reanimation, Ankara University, Faculty of Medicine, Ankara, Turkey, and ${ }^{4}$ Department of Biochemistry and Clinic Biochemistry, Firat University Faculty of Medicine, Elazig, Turkey

Address for correspondence: A.B. Ozer, Department of Anesthesiology and Reanimation, Firat University Medical School, Department of Anesthesiology and Reanimation, 23119, Elazig, Turkey.

Phone/Fax: +90.533.4478924

Funding: Firat University Scientific Research Projects Unit. neurotransmission, while in mature brains it occurs by a decrease in GABAergic neurotransmission (6, 7).

Although general anaesthesia is considered safe, experimental studies have shown that it might have harmful effects on the developing mammalian brain $(8,9)$. It was also shown that exposure to anaesthetics that antagonise N-methyl-D-aspartate (NMDA) receptors or stimulate GABA-A receptors during synaptogenesis in the developing brain may trigger common apoptotic neurodegeneration (10).

The current study was planned to detect an acute phase brain damage that occurs due to the anaesthetics commonly used in pediatric patient population, to investigate the effects of anaesthesia on long-term behaviour, anxiety and impact on learning as well as to determine whether there is a correlation between these effects and serum BDNF, brain BDNF, amino acid levels and apoptosis.

\section{Materials and methods}

The preclinical animal study was conducted at the Firat University Experimental Research Centre in December 2012. After receiving approval from the institutional Animal Experimentation Ethics Committee, seven-day-old male Wistar-Albino rats were obtained from the Experimental Research Centre. The Helsinki Universal Declaration of Animal Rights was followed at every stage of the study. The rats were kept in the rooms with ambient temperature of $22-24^{\circ} \mathrm{C}$ and with $12 / 12$ hour day/night cycle. Except for the time it took for the experimental tests, the subjects 


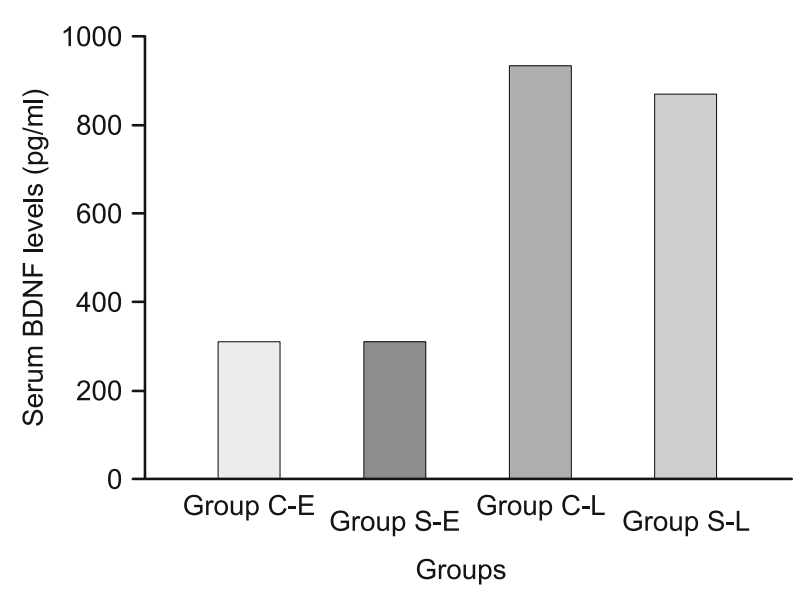

Fig. 1. Serum brain-derived-neurotrophic factor (BDNF) levels of groups.

were kept in the same cage with their mothers until postnatal day 21 (PN21). After the 21st day, the rats were put in separate cages and fed with standard rat chow and tap water.

Using permuted block randomisation methods, the subjects were divided into the two groups: Group $\mathrm{C}$ acted as the control and did not receive any anaesthesia, and in Group S, anaesthesia was achieved with $2.3 \%$ sevoflurane in $50 \%$ oxygen $\left(\mathrm{O}_{2}\right)$-air mixture. The concentration of sevoflurane was adjusted according to the tail test. The tail test was applied every 15 minutes. The middle $1 / 3$ of the tail was clamped, and if there was response, sevoflurane concentration was increased $15 \%$. All subjects were put in a plastic, transparent anaesthesia chamber that was connected to the anaesthesia device and was ventilated with $4 \mathrm{~L} / \mathrm{min}$ flow and $50 \% \mathrm{O}_{2}$-air mixture. Immediately after the six-hour administration of anaesthesia, oxygen arterial blood gases were evaluated in 3 subjects from each group. At the end of the application period, half of the subjects were sacrificed to determine the early effects of sevoflurane (Group SE and Group CE), while the rest of the subjects were sacrificed 6 weeks after the application to determine the late effects of sevoflurane (Group SL and Group CL). In addition, the levels of serum BDNF, brain tissue BDNF and caspase 3 were evaluated for all subjects. The anaesthesia chamber was heated from the outside during the entire experiment to prevent hypothermia. Glucose and saline were administered intradermally to prevent hypoglycaemia and hypovolaemia. Subjects that experienced discolouration of the skin (cyanosis) or a decrease in respiratory rate that did not improve with stimuli, were excluded from the study.

Tab. 1. Brain-derived-neurotrophic factor (BDNF) and caspase 3 levels in cortex and hippocampus (median [min-max]).

\begin{tabular}{lcccc}
\hline & $\begin{array}{c}\text { BDNF } \\
\text { cortex }\end{array}$ & $\begin{array}{c}\text { BDNF } \\
\text { hippocampus }\end{array}$ & $\begin{array}{c}\text { Caspase 3 } \\
\text { cortex }\end{array}$ & $\begin{array}{c}\text { Caspase 3 } \\
\text { hippocampus }\end{array}$ \\
\hline Group CE & $1.5(1-3)$ & $1.5(0-3)$ & $0.5(0-1)$ & $0.5(0-1)$ \\
Group SE & $1.5(0-2)$ & $1.5(1-2)$ & $2(1-2)$ & $1(0-1)$ \\
Group CL & $1(0-1)$ & $1(0-2)$ & $0(0-1)$ & $0.5(0-1)$ \\
Group SL & $0.5(0-1)$ & $1(0-2)$ & $1(1-2)$ & $1(0-2)$ \\
\hline
\end{tabular}

The study's primary outcomes were serum BDNF levels, cortex and hippocampal BDNF levels and neurocognitive status. Secondary outcomes were cortex and hippocampal caspase 3 levels. Blood samples were taken at decapitation phase into serum separator tube to evaluate serum BDNF. Blood samples were centrifuged at $1000 \mathrm{x}$ g for 15 minute and stored at $-20^{\circ} \mathrm{C}$. Serum BDNF levels were measured by the enzyme-linked immunosorbent assay (ELISA) method (EK0308, Boster Biological Technology, Ltd.). Brain tissue samples were kept for 24 hours in $10 \%$ formaldehyde prepared with phosphate buffer saline. Brain tissue samples were taken for routine tissue processing for immunohistochemical (IHC) examination procedure following the sagittal reduction process. Brain tissue was divided at the midline on the sagittal plane. The BDNF levels (Abcam, ab108319, Cambridge, UK) and caspase 3 levels (Abcam, ab13847, Cambridge, UK) were assessed with IHC in brain hemispheres (0 - no staining, 1 - mild, 2 - moderate, 3 - severe).

The behaviour, anxiety states and spatial learning abilities of the subjects during the long-term period (6 weeks later) were evaluated by using the plus arm test and the Morris water test, respectively. Open arm avoidance index was calculated according to the formula $100-((\%$ of the time spent in the open arms + $\%$ of the entrance to the open arms)/2), while the total locomotor activity was calculated based on line crossings + rearing. Swimming tests were done 4 times a day for a period of 4 days. The test was repeated 2 days after training and the time it took to reach the platform (latency) and the time spent in the platform quadrant after the platform was removed were recorded. The subjects that completed the swimming test were put back into their heated cages.

Those conducting the experiment knew, which group of subjects they were dealing with, but those evaluating biochemical, IHC and neurocognitive tests did not know, which samples and subjects belonged to which group.

SPSS 15 was used for a statistical evaluation. Nonparametric methods were used for all variables because the sample size was small. Kruskal-Wallis test was used for one-way analysis of variance (ANOVA) of nonparametric data, therefore median values were calculated instead of mean. When it was determined not to the equal of medians with Kruskal-Wallis test, Mann-Whitney U test was used for post-hoc multiple comparisons. The escape latency within the group was evaluated by Wilcoxon test. The correlation between parameters was assessed by Spearman correlation test and $\mathrm{p}<0.05$ was considered significant.

\section{Results}

The 30 rats in the study were divided into groups $\mathrm{C}$ and $\mathrm{S}$ of 15 (50 \%) each that were further divided into the early and late subgroups. BDNF levels in the late period groups were higher than in the early period groups $(p<0.05)$ (Fig. 1). While there was no significant difference in the BDNF levels in the cerebral hippocampus, the BDNF levels in cerebral cortex were higher in the Group CE than the Group CL and the Group SL (p < 0.05) (Tab. 1 ). In cerebral cortex of all rats, most significant release of BDNF was present in large neurons in the outer granular layer and the 

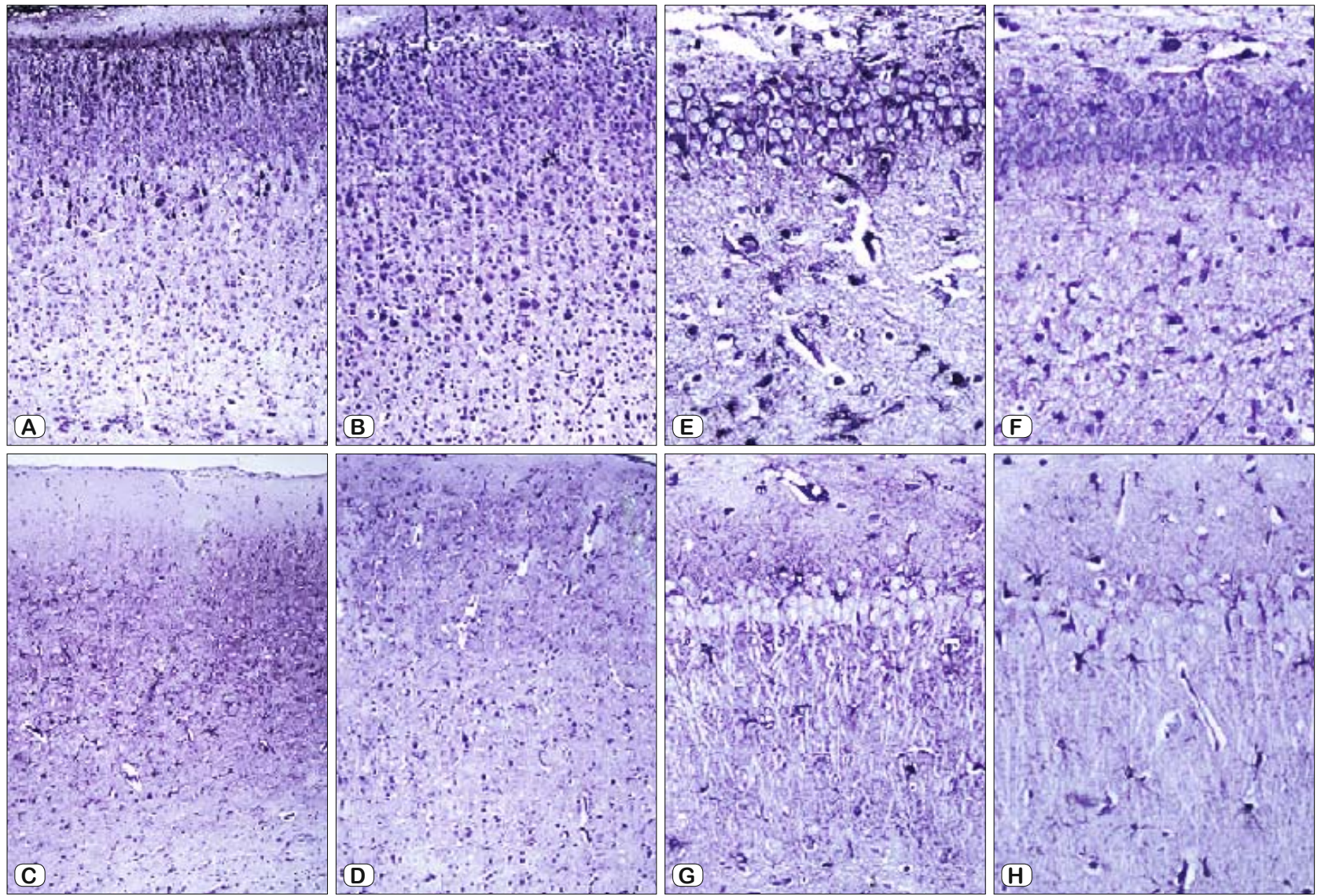

Fig. 2. Brain-derived-neurotrophic factor (BDNF) immunohistochemistry staining in the cortex (200 $\mu \mathrm{m})(\mathrm{A}-\mathrm{Group}$ CE, B - Group SE, E Group CL, F - Group SL); in the hippocampus (C - Group CE, D - Group SE, G - Group CL, H - Group SL).

outer pyramidal layer. Release of BDNF was detected in all layers of neocortex. Between all groups, the release of BDNF in multiformed layer was prominent in the Group CE (Fig. 2). There was a significant negative correlation between serum BDNF levels and the cortex BDNF levels ( $p=0.003 ; r=-0.425$ ). Cortex caspase 3 levels were significantly higher in the Groups SE and SL than in the Group CE and the Group CL, especially outer granulary, outer pyramidal layer and pyramidal layer $(\mathrm{p}<0.05)$ (Fig. 3).

There was no significant difference between the groups in terms of open arm index and locomotor activity $(p>0.05)$. Fourday and last-day Morris water test showed no significant difference between groups in terms of time to reach the platform $(p>0.05)$. There was a negative correlation between serum BDNF and time to reach the platform ( $p=0.022, r=-0.509)$. It was observed that in all groups the time to reach the platform became shorter with time ( $<<0.05)$. When the platform was removed, the time spent in the quadrant with the platform was not significantly different between the groups ( $p>0.05)$.

\section{Discussion}

Sevoflurane is the most commonly used inhalational anaesthetic for pediatric surgical cases due to its minimal airway reactivity and low blood/gas partition coefficient. Therefore, we evaluated sevoflurane's effect on the brain of neonatal rat in our study. We have found that serum levels of BDNF increased and brain levels decreased over time. There could be two explanations. Firstly, serum BDNF levels are known to increase with age (11). Secondly, it has been reported that hypoxia increases BDNF levels and that continuous positive airway pressure (CPAP) treatment decreases BDNF levels (12-14). In our study, seven-day-old rats were exposed to oxygen for 6 hours. Therefore, BDNF levels may also be measured so low in the early stages.

While low doses of ketamine did not result in changes in hippocampal BDNF levels, higher doses were shown to increase them (15). Nine hours of $20 \mathrm{mg} / \mathrm{kg}$ ketamine treatment was reported to increase total BDNF protein levels in the brain (16). In 10-day-old mice 24 hours after propofol administration, BDNF protein levels were reported to decrease in the parietal cortex, but increased in the frontal cortex and hippocampus (17). The BDNF levels in the hippocampus were increased by ketamine, but remained unchanged when treated with propofol alone or in combination with ketamine (18). Yu et al evaluated BDNF levels in hippocampal cornus ammonis 3 (CA3) area of seven-dayold that were exposed to $3.6 \%$ sevoflurane during six hours at postnatal 21 days (19). It demonstrated no significant differences 

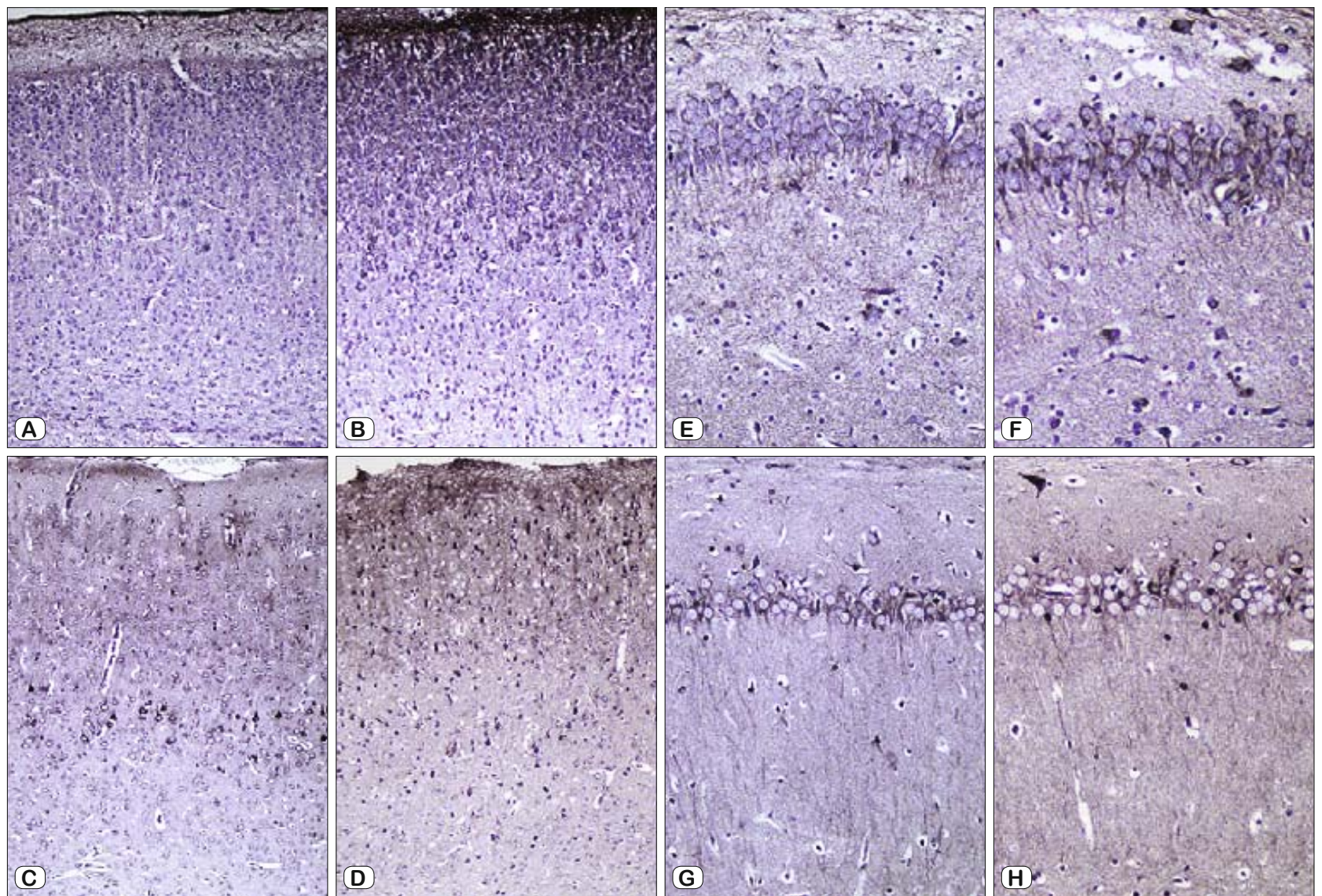

Fig. 3. Caspase 3 immunohistochemistry staining in the cortex $(200 \mu \mathrm{m})(\mathrm{A}-$ Group $\mathrm{CE}, \mathrm{B}$ - Group SE, E - Group CL, F - Group SL); in the hippocampus (C - Group CE, D - Group SE, G - Group CL, H - Group SL).

between sevoflurane group and control group, when BDNF levels were evaluated with IHC. However, BDNF messenger ribonucleic acid (mRNA) expression, evaluated by reverse transcription polymerase chain reaction (RT-PCR) and BDNF protein level was evaluated by Western Blot, decreased in sevoflurane group (19). In our study, we found that BDNF levels were not different between the groups. The study evaluated effects of sequential exposures to sevoflurane on hippocampal BDNF levels of neonatal rats (postnatal six, seven and eight days). On postnatal 21 day, the subjects were divided into the two groups according to different environments. While BDNF levels decreased in the only remaining subjects in the cage, they did not change in pair subjects in the cage (20). In our study, BDNF levels did not decrease because the subjects were not left alone in a cage except for the experimental protocol. In our study, the cerebral cortex BDNF levels were higher in the Group CE than in the Group CL and the Group SL.

In studies with seven-day-old rats where loss of pyramidal neurons was induced, levels of caspase 3 were increased in the hippocampus upon administration of sevoflurane, propofol and ketamine (17, 21-24). In our study, cortex caspase 3 levels were found to be significantly higher in the sevoflurane group compared to the control group.
In our study, there was no significant difference between the groups in terms of open-arm avoidance index and locomotor activity. In the study, where sevoflurane was applied for six hours to seven-day-old rats, the battery fox test did not cause any significant changes (25). In our study, there was no significant difference between the groups in terms of time to reach the platform in the Morris water maze (MWM) test. When the platform was removed, the time subjects spent in the platform quadrant was not significantly different in Group SL compared to Group CL. There was no significant difference in the MWM test of the seven-day-old rats treated with sevoflurane for six hours (25). Moreover, the administration of a high concentration of isoflurane during the intrauterine period resulted in a decrease in the time spent to reach the platform in MWM test in neonatal rats, but this time was not significantly different when isoflurane was administered at low concentrations (26). In the study where sevoflurane was applied for six hours to seven-day-old rats, latency duration of MWM test increased (19). Differences of this study from our study are the applied sevoflurane dose (3.6 \% to 2.3 $\%)$ and timing of MWM test (PN21 to PN49-53). Zhang et al demonstrated no differences in terms of neuro-behavioural tests in the subjects that were provided the same conditions as in our study (20). 
80-84

The major limitation of our study was the small number of subjects. For the statement "sevoflurane might be a reliable anaesthetic in a pediatric patient”, more detailed studies are needed.

Serum BDNF levels increased over time, but the opposite was found to be correlated with brain BDNF levels. Sevoflurane did not cause significant changes in the serum, cerebral cortex and hippocampus BDNF levels, but significantly more in the cortex. Sevoflurane caused more apoptosis. Sevoflurane did not cause a reduction in spatial learning and anxiety. And, although sevoflurane induced apoptosis, it did not affect the levels of BDNF and showed no long-term negative effects on learning and anxiety in neonatal rats.

\section{Learning point}

Anaesthesia is applied to millions of children for surgery, imaging and other invasive procedures, the issue is very serious and concerns. Sevoflurane, is the most used inhalation anaesthetic, which does not affect spatial learning and anxiety, even if it increases neuro-apoptosis.

\section{References}

1. Acheson A, Conover JC, Fandl JP et al. A BDNF autocrine loop in adult sensory neurons prevents cell death. Nature 1995; 374: 450-453

2. Huang EJ, Reichardt LF. Neurotrophins: roles in neuronal development and function. Annu Rev Neurosci 2001; 24: 677-736.

3. Yamada K, Nabeshima T. Brain-derived neurotrophic factor/TrkB signaling in memory processes. J Pharmacol Sci 2003; 91: 267-270.

4. Baldelli P, Novara M, Carabelli V, Hernandez-Guijo JM, Carbone E. BDNF up-regulates evoked GABAergic transmission in developing hippocampus by potentiating presynaptic N- and P/Q-type Ca2+ channels signalling. Eur J Neurosci 2002; 16: 2297-2310.

5. Sherwood NT, Lo DC. Long-term enhancement of central synaptic transmission by chronic brain-derived neurotrophic factor treatment. J Neurosci 1999; 19: 7025-7036.

6. Kojima M, Klein RL, Hatanaka H. Pre- and post-synaptic modification by neurotrophins. Neurosci Res 2002; 43: 193-199

7. Tanaka T, Saito H, Matsuki N. Inhibition of GABAA synaptic responses by brain-derived neurotrophic factor (BDNF) in rat hippocampus. J Neurosci 1997; 17: 2959-2966.

8. Cote CJ. Pediatric Anesthesia. In: Miller RD, ed. Miller's Anesthesia, Philadelphia: Elsevier Churchill Livingstone, 2005; 2367-408.

9. Hayashi H, Dikkes P, Soriano SG. Repeated administration of ketamine may lead to neuronal degeneration in the developing rat brain. Paediatr Anaesth 2002; 12: 770-774.

10. Ikonomidou C, Bittgau P, Koch $\mathbf{C}$ et al. Neurotransmitters and apoptosis in the developing brain. Biochem Pharmacol 2001; 62: 401-405.

11. Imam SS, Gad GI, Atef SH, Shawky MA. Cord blood brain derived neu-rotrophic factor: diagnostic and prognostic marker in fullterm newborns with perinatal asphyxia. Pak J Biol Sci 2009; 12: 1498-1504.
12. Scheepens A, Wassink G, Blanco CE. The effect of a global birth asphyxia on the ontogeny of BDNF and NGF protein expression in the juvenile brain. Brain Res Dev Brain Res 2003; 140: 215-221.

13. Fleiss B, Coleman HA, Castillo-Melendez M, Ireland Z, Walker DW, Parkington HC. Effects of birth asphyxia on neonatal hippocampal structure and function in the spiny mouse. Int J Dev Neurosci 2011; 29: 757-766.

14. Korhonen L, Riikonen R, Nawa H, Lindholm D. Brain derived neurotrophic factor is increased in cerebrospinal fluid of children suffering from asphyxia. Neurosci Lett 1998; 240: 151-154.

15. Garcia LS, Comim CM, Valvassori SS et al. Acute administration of ketamine induces antidepressant-like effects in the forced swimming test and increases BDNF levels in the rat hippocampus. Prog Neuropsychopharmacol Biol Psychiatry 2008; 32: 140-144.

16. Ibla JC, Hayashi H, Bajic D, Soriano SG. Prolonged exposure to ketamine increases brain derived neurotrophic factor levels in developing rat brains. Curr Drug Saf. 2009; 4: 11-16.

17. Kong FJ, Ma LL, Hu WW, Wang WN, Lu HS, Chen SP. Fetal exposure to high isoflurane concentration induces postnatal memory and learning deficits in rats. Biochem Pharmacol 2012; 84: 558-563.

18. Kohara K, Kitamura A, Adachi N et al. Inhibitory but not excitatory cortical neurons require presynaptic brain-derived neurotrophic factor for dendritic development, as revealed by chimera cell culture. J Neurosci 2003; 23: 6123-6131.

19. Yu Z, You S, Xu M, Lin H, Di MQ, Lian QQ. Effect of acute exposure to sevoflurane and isoflurane on learning, memory and brain derived neurotrophic factor expression in hippocampus of juvenile SD rats. Chin J Pharmacol Toxicol 2013; 27: 132-137.

20. Zhang MQ, Ji MH, Zhao QS et al. Neurobehavioural abnormalities induced by repeated exposure of neonatal rats to sevoflurane can be aggravated by social isolation and enrichment deprivation initiated after exposure to the anaesthetic. Br J Anaesth 2015; 115: 752-760.

21. Soriano SG, Liu Q, Li J et al. Ketamine activates cell cycle signaling and apoptosis in the neonatal rat brain. Anesthesiology 2010; 112: 1155-1163.

22. Yu D, Jiang Y, Gao J, Liu B, Chen P. Repeated exposure to propofol potentiates neuroapoptosis and long-term behavioral deficits in neonatal rats. Neurosci Lett 2013; 534: 41-46.

23. Liu F, Paule MG, Ali S, Wang C. Ketamine-induced neurotoxicity and changes in gene expression in the developing rat brain. Curr Neuropharmacol 2011; 9: 256-261.

24. Zou X, Patterson TA, Sadovova N et al. Potential neurotoxicity of ketamine in the developing rat brain. Toxicol Sci 2009; 108: 149-158.

25. Feng X, Liu JJ, Zhou X et al. Single sevoflurane exposure decreases neuronal nitric oxide synthase levels in the hippocampus of developing rats. Br J Anaesth 2012; 109: 225-233

26. Wang JH, Fu Y, Wilson FA, Ma YY. Ketamine affects memory consolidation: differential effects in T-maze and passive avoidance paradigms in mice. Neurosci 2006; 140: 993-1002.

Received October 2, 2016. Accepted November 18, 2016. 